erschweren wird. Zudem stellt die in COMPLEMENT 1 verwendete Chlorambucil-Monotherapie aus heutiger Sicht sicherlich keinen adäquaten Vergleichsarm mehr dar. Die aktuelle Verfügbarkeit von 3 verschiedenen CD20-Antikörpern in der Erstlinientherapie führt nun unweigerlich zu indirekten Vergleichen zwischen den entsprechenden großen klinischen Studien, vor allem zwischen CLL11 und COMPLEMENT 1. Formell ist dies sicherlich nicht schlüssig möglich, da sich beide Studien unterscheiden - sowohl hinsichtlich der Patientencharakteristika und der Chlorambucil-Dosierung als auch in Bezug auf die verwendete Methodik und das Follow-up. Dennoch fällt auf: In der CLL11-Studie war die Obinutuzumab-haltige Immunchemotherapie gegenüber Chlorambucil mono mit einem signifikanten OS-Vorteil verbunden; in den Daten der COMPLEMENT 1-Studie zeichnet sich ein solcher Vorteil für Ofatumumab plus Chlorambucil bisher nicht ab. Andererseits scheint das Nebenwirkungsspektrum für Ofatumumab tendenziell eher günstiger zu sein, insbesondere was Infusionsreaktionen und Neutropenien betrifft.

Abgesehen vom "head-to-head"-Vergleich von Obinutuzumab mit Rituximab in der CLL11-Studie ist bisher keine abschließende vergleichende Beurteilung von Wirksamkeits- und Verträglichkeitsunterschieden der verfügbaren CD20-Antikörper möglich. Zudem ist die optimale Chlorambucil-Dosierung in Kombination mit einem CD20-Antikörper weiterhin unklar. Die Ergebnisse der COMPLEMENT 1-Studie bedeuten einen weiteren großen Schritt in Richtung einer effektiven Erstlinientherapie auch bei älteren und unfitten Patienten. Die guten Daten für Ansprechen und PFS bestätigen und festigen damit die Basis für die Immunche- motherapie als aktuellen Behandlungsstandard bei diesem Patientenkollektiv. Somit wird die Immunchemotherapie auch zum neuen Standardarm für Vergleiche mit zukünftigen Therapieregimes, die ggf. ohne Chlorambucil als "backbone" auskommen; Ziel solcher Studien wird es sein, neuere zielgerichtete Substanzen (wie z. B. Ibrutinib, Idelalisib oder in absehbarer Zukunft auch Venetoclax) alleine oder in Kombination in Bezug auf Effektivität und Toxizität in der Erstlinienbehandlung zu überprüfen.

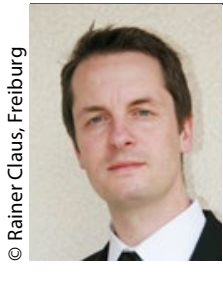

PD Dr. med Rainer Claus Abteilung für Hämatologie, Onkologie und Stammzelltransplantation, Universitätsklinikum Freiburg rainer.claus@uniklinikfreiburg.de

\section{Risikoscore für Patienten mit fortgeschrittener Mycosis fungoides und Sézary-Syndrom}

\section{Prognose und Risikofaktoren von Mycosis fungoides und Sézary-Syndrom wurden bisher meist monozentrisch analysiert, was angesichts der Seltenheit beider Erkrankungen nur zu vorläufigen Ergebnissen führen konnte. Nun ist ein großes internationales Konsortium diesen Fragen auf Basis größerer Patientenzahlen nachgegangen.}

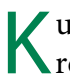
utane T-Zell-Lymphome präsentieren sich meist als Mykosis fungoides (MF) und seltener als Sézary-Syndrom (SS). Während frühe Stadien vergleichsweise günstig verlaufen, verhalten sich die Stadien IIB-IVB aggressiv und haben eine schlechtere Prognose. Ein Drittel der Patienten präsentiert sich mit fortgeschrittenem Haut- (T3-T4) und/ oder nodalem Befall. In einem internationalen Cutaneous Lymphoma International Consortium (CLIC) sollte nun multizentrisch die Prognose beider Erkrankungen in Abhängigkeit vom Stadium geprüft und der Stellenwert verschiedener prognostischer Marker bestimmt werden. Neben dem Stadium wurden neun Marker auf ihren Einfluss auf das Überleben von MF/SS-Patienten untersucht: Alter, Geschlecht, histologische Zeichen von Follikulotropismus, CD30Positivität, Proliferationsindex, großzel- lige Transformation, Leukozyten-/Lymphozyten-Zahl, LDH und der Nachweis identischer Zellklone im Blut.

Für 1.275 Patienten aus 29 Zentren mit MF/SS der Stadien $\geq$ IIB lag das mediane Überleben bei 63 Monaten mit 2- und 5-Jahres-Überlebensraten von $77 \%$ bzw. $52 \%$. Im Stadium IIB überlebten nach einem, drei und fünf Jahren $88,5 \%$, $80,1 \%$ und 57,4\%. Die Ergebnisse lagen für die Stadien III insgesamt sowie für IIIA und IIIB in demselben Bereich, wohingegen sich die Prognose im Stadium IVA und IVB mit medianen Überlebenszeiten von 47,5 bzw. 33,3 Monaten drastisch verschlechterte (5-Jahres-Überlebensraten $42,9 \%$ und 39,0\%). Nur in der univariaten Analyse hatten Lymphome mit Follikulotropismus eine bessere $(\mathrm{p}<0,001)$ und Patienten mit Leukozytose eine schlechtere Prognose $(p=0,006)$. Von den insgesamt untersuchten zehn
Variablen hatten vier in der uni- wie in der multivariaten Analyse einen signifikanten Einfluss auf das Überleben: Stadium IV, Alter > 60 Jahre, großzellige Transformation und erhöhte LDH.

Als unabhängige prognostische Marker für schlechteres Überleben wurden diese vier zur Erstellung eines prognostischen Index genutzt und die Patienten Risikogruppen zugeordnet: niedriges Risiko 0-1 positive Variablen, intermediär bei 2 Variablen und hoch bei 3-4 Risikofaktoren. Entsprechend dieser Zuordnung lassen sich signifikante Unterschiede in den Überlebenskurven ( $p$ jeweils $<0,001)$ nachweisen. Die 5-JahresÜberlebensraten fallen von $68 \%$ bei niedrigem Risiko über $44 \%$ (intermediär) auf $28 \%$ in der Hochrisikogruppe ab.

Fazit: Mit dieser bisher größten Kohorte von Patienten mit fortgeschrittenenr MF/SS konnte ein prognostischer Index gebildet werden, der für die Risikoabschätzung und Stratifizierung individueller MF/SS-Patienten genutzt werden kann.

Brigitte Schalhorn

Scarisbrick JJ et al. Cutaneous Lymphoma International Consortium Study of Outcome in Advanced Stages of Mycosis Fungoides and Sézary Syndrome: Effect of Specific Prognostic Markers on Survival and Development of a Prognostic Model. J Clin Oncol. 2015;33(32):3766-73. 